\title{
Unique microRNA profile in Dupuytren's contracture supports deregulation of $\beta$-catenin pathway
}

\author{
Neda Mosakhani ${ }^{1, *}$, Mohamed Guled ${ }^{1, *}$, Leo Lahti ${ }^{1,2}$, Ioana Borze ${ }^{1}$, Minna Forsman $^{3}$, \\ Virve Pääkkönen ${ }^{4}$, Jorma Ryhänen ${ }^{3}$ and Sakari Knuutila ${ }^{1}$ \\ ${ }^{1}$ Department of Pathology, Haartman Institute and HUSLAB, University of Helsinki and Helsinki University \\ Central Hospital, Helsinki, Finland; ${ }^{2}$ Department of Information and Computer Science, Aalto University \\ School of Science and Technology, Espoo, Finland; ${ }^{3}$ Department of Surgery, University of Oulu, Oulu, \\ Finland and ${ }^{4}$ Institute of Dentistry, University of Oulu, Oulu, Finland
}

\begin{abstract}
Dupuytren's contracture, a proliferative disease of unknown origin, is characterized by an abnormal fibroblast proliferation process. Evidence from numerous microRNA (miRNA) studies shows that miRNAs have a vital function in many biological processes, for instance, in cellular signaling networks, cell growth, tissue differentiation, and cell proliferation. Our aim was to characterize, to our knowledge for the first time, the miRNA-expression profile of Dupuytren's contracture. The miRNAs identified may have a function in the pathogenesis of Dupuytren's contracture by targeting and regulating important pathways. We compared the miRNA-expression profile of 29 Dupuytren's contracture patients with that of control samples (fibroblast cells and palmar fascia). Some of the miRNAs identified in our Dupuytren's contracture samples, including miR-29c, miR-130b, miR-101, miR-30b, and miR-140-3p, were found to regulate important genes related to the $\beta$-catenin pathway: WNT5A, ZIC1, and TGFB1. Expression profiles of these genes reanalyzed from published gene-expression data from similar patient material correlated with our miRNA results. Analysis was also performed for groups of patients with recurrent/non-recurrent and patients with hereditary/non-hereditary Dupuytren's contracture, but no significant differences appeared in miRNA-expression profiles of these groups. Identification of unique miRNA expression in Dupuytren's contracture may lead to the development of novel molecular therapy for its treatment.

Modern Pathology (2010) 23, 1544-1552; doi:10.1038/modpathol.2010.146; published online 30 July 2010
\end{abstract}

Keywords: Dupytren's contracture; microarray; miRNA

Dupuytren's contracture is a benign disease with an abnormal fibroblast proliferation process and unclear pathogenesis. ${ }^{1}$

Epidemiological reports show that this condition predominantly affects older men of northern European descent, but it is also common in the Taiwanese population and in some areas of Japan, although deformity is less severe in the latter two areas. ${ }^{2}$ Prevalence between genders, however, tends to equalize toward the ninth decade of life. ${ }^{3}$

Correspondence: Professor S Knuutila, PhD, Department of Pathology, Haartman Institute, PO Box 21 (Haartmaninkatu 3), FI-00014 University of Helsinki, Helsinki, Finland.

E-mail: sakari.knuutila@helsinki.fi

*These two authors contributed equally to this work.

Received 27 April 2010; revised 1 June 2010; accepted 7 June 2010; published online 30 July 2010
Dupuytren's contracture is associated with smoking and diabetes mellitus, ${ }^{2}$ with the mechanisms behind these associations still unknown. It is, however, likely that smoking causes microvascular changes in the hand, and in diabetes patients, microangiopathy and increased collagen production may contribute to its development. ${ }^{4}$

One predisposing factor is genetic susceptibility. ${ }^{5}$ One study has suggested an associated heteroplasmic mitochondrial mutation. ${ }^{6}$ Another report shows autosomal dominant inheritance with incomplete penetrance at $16 \mathrm{q} .^{7}$

Several studies have investigated the molecular pathogenesis of Dupuytren's contracture. These include gene-expression profiling,,$^{5,8-12}$ cytogenetic profiling, ${ }^{13-16}$ and proteomic profiling. ${ }^{17}$ These studies have identified genes that may potentially be involved in its pathogenesis. 
MicroRNAs (miRNAs) are small, single-stranded non-coding endogenous RNAs consisting of 20-23 nucleotides. ${ }^{18,19}$ They have been implicated in $>70$ disease $^{20}$ including diabetes mellitus, asthma, hepatitis, and a variety of cancers (http://cmbi.bjmu. edu.cn/hmdd).

This study investigates differences in miRNA expression between Dupuytren's contracture patient samples and normal controls, including cultured fibroblast cells and palmar fascia. Differentially expressed miRNAs could potentially have a function in the underlying mechanisms of its pathogenesis by dysregulating Dupuytren's contracture-associated genes. The mRNA-expression profiles of Dupuytren's contracture-associated genes, which are among the target genes of differentially expressed miRNAs found in this study, were reanalyzed to confirm whether there exists any correlation between miRNA and their target gene expression.

\section{Materials and methods}

\section{Patients}

We used 29 fresh frozen samples of removed cords from patients (see Table 1) diagnosed with Dupuytren's contracture. No patient had knuckle pads, Peyronie's, or Ledderhose's diseases. Patients were preselected before undergoing aponeurectomy to remove the extension limitation of 30 degrees in the metacarpophalangeal joint or to heal the midpalmar cord interfering with normal functions of the palm. Only the disabling cords (which develop from the matured nodules organized in parallel lines), not the individual nodules, were operated on. Fasciectomy was performed for these patients, removing the entire affected palmar fascia. The skin was opened with $\mathrm{Z}$ plastia to allow sufficient skin for closure, but no skin was removed. Two patients needed skin grafts for the closure. ${ }^{17,18}$

According to the standard surgical procedure, two-hand surgeons clinically estimated and evaluated each patient. Clinically, all the excised samples were in the mature phase of the Dupuytren's contracture, and no histological evaluation was performed pre-operatively. Six cases were recurrent among our patients. The definition for recurrence is still entirely a clinical diagnosis. After aponeurectomy (excision of the Dupuytren cord), within a few years, a new palpable nodule or cord gradually appears, which causes limitation of finger extension. The time of the recurrence for our samples was at least 2-5 years. After surgery, the samples were maintained in $\mathrm{N}_{2}$ until RNA extraction. Of the 29 samples, we studied 17 for gene copy number changes using an oligonucleotide-array CGH method as previously described. ${ }^{16}$

We used standard cell cultured fibroblasts (ATCC cell line: CRL-2088) and normal palmar fascia as control samples from which we extracted total RNA. Although it is not easy to acquire normal healthy palmar fascia, considering availability and ethical issues, a small number of control palmar fascias were obtainable from two healthy, non-smoking males with hand trauma (age 25 and 46 years) and two females with median nerveneurolysis in the carpal tunnel, the carpal tunnel fascia being the transverse ligament beneath the actual palmar fascia. One woman was a non-smoker with cardiac inefficiency and bronchial asthma, age 85 years, and the other a smoker with a healthy status, age 37 years.

\section{RNA Extraction}

Qiagen's miRNeasy Mini kit (Qiagen, Valencia, CA, USA) was used to extract total RNA, including miRNA, according to manufacturer's instructions. We used Agilent's miRNA Microarray system (V2), containing 723 human and 76 human viral miRNAs catalogued in the Sanger miRNA database v 10 (Agilent Technologies, Santa Clara, CA, USA). The NanoDrop-1000 Spectrophotometer (Thermo Fisher Scientific, Wilmington, DE, USA) was used for quantification of RNA and Agilent Bioanalyzer for a quality check of both RNA and miRNA.

\section{Labeling, Hybridization, Scanning, and Data Processing}

Agilent's miRNA microarray platform has been shown by Wang et $a l^{21}$ to produce precise and accurate measurements of miRNA expression. Labeling and hybridization of RNA samples were performed with the Agilent's miRNA Complete Labeling and Hybridization kit. Accordingly, $100 \mathrm{ng}$ of total RNA was treated with calf intestine phosphatase for $30 \mathrm{~min}$ at $37^{\circ} \mathrm{C} ; 100 \%$ DMSO was used for denaturation at $100^{\circ} \mathrm{C}$ for $5 \mathrm{~min}$, after which the samples were immediately transferred into an ice water bath to prevent re-annealing. Samples were then labeled with cyanine 3-pCp by incubation with T4 RNA ligase for $2 \mathrm{~h}$ at $16^{\circ} \mathrm{C}$. After the labeling reaction, the samples were vacuum dried at medium heat and resuspended in nuclease-free water. Next, the samples were hybridized to the microarrays in the Agilent SureHyb Chambers (Agilent Technologies) for $20 \mathrm{~h}$ at $55^{\circ} \mathrm{C}$. The microarrays were then washed with the manufacturer's washing buffers. Arrays were scanned with the Agilent's Scanner, and the raw data was reprocessed with the Agilent's Feature Extraction Software with default parameters. Details of the miRNA preprocessing protocol are provided in the manufacturer's reference guide for the Agilent Feature Extraction tool.

The statistical analysis was carried out with the GeneSpring GX Analysis Software v 10, (Agilent Technologies) and the $\mathrm{R}$ statistical programming language (http://www.r-project.org). The expression values were $\log _{2}$ transformed before the analysis. The miRNAs that were not detected in any of the 
Table 1 Clinical features of Dupuytren's contracture patients

\begin{tabular}{|c|c|c|c|c|c|c|c|c|c|c|}
\hline Sample & Sex & Smoking & Recurrence & Hereditary & $\begin{array}{l}\text { Patient } \\
\text { observation }\end{array}$ & $\begin{array}{l}\text { Age at } \\
\text { surgery }\end{array}$ & $\begin{array}{c}\text { Bilateral } \\
\text { involvment }\end{array}$ & $\begin{array}{c}\text { Radial ray } \\
\text { involvment } \\
\text { (first or second) }\end{array}$ & $\begin{array}{l}\text { Little finger } \\
\text { surgery }\end{array}$ & $\begin{array}{c}\text { Score of } \\
\text { Abe et al }{ }^{46}\end{array}$ \\
\hline 1 & $\mathrm{~F}$ & No & No & No & HTA, MC & 67 & + & & & 3 \\
\hline 2 & $\mathrm{M}$ & No & No & No & Healthy & 61 & & & + & 1 \\
\hline 3 & $\mathrm{M}$ & No & No & No & HTA & 65 & & & & 0 \\
\hline 4 & $\mathrm{M}$ & No & No & Yes & HC, HTA & 70 & + & & + & 2 \\
\hline 5 & M & No & Yes & No & Healthy & 70 & & + & + & 3 \\
\hline 6 & $\mathrm{M}$ & No & No & No & Healthy & 72 & & & + & 1 \\
\hline 7 & $\mathrm{M}$ & No & No & No & Healthy & 56 & + & & & 1 \\
\hline 8 & $\mathrm{M}$ & No & yes & No & Healthy & 64 & & & + & 1 \\
\hline 9 & M & No & No & No & $\mathrm{AB}, \mathrm{HC}, \mathrm{DM}$ & 71 & + & & & 1 \\
\hline 10 & $\mathrm{M}$ & No & No & No & Healthy & 77 & & & & 0 \\
\hline 11 & $\mathrm{M}$ & No & No & No & Healthy & 80 & & & + & 1 \\
\hline 12 & $\mathrm{M}$ & No & No & No & CMTD, MBM & 61 & & & + & 1 \\
\hline 13 & $\mathrm{M}$ & Yes & No & No & $\mathrm{CD}, \mathrm{HC}$ & 50 & & & & 1 \\
\hline 14 & $\mathrm{M}$ & No & Yes & No & Healthy & 57 & + & & + & 2 \\
\hline 15 & $\mathrm{M}$ & Yes & No & No & RS & 67 & + & & + & 2 \\
\hline 16 & $\mathrm{M}$ & No & No & Yes & Healthy & 51 & + & & + & 2 \\
\hline 17 & M & No & No & Yes & Healthy & 66 & + & + & + & 4 \\
\hline 18 & $\mathrm{M}$ & No & Yes & Yes & Healthy & 67 & + & + & + & 4 \\
\hline 19 & $\mathrm{M}$ & Yes & No & No & HTA & 80 & & & & 0 \\
\hline 20 & M & Yes & No & Yes & Arrhythmia & 59 & & & & 0 \\
\hline 21 & M & No & No & Yes & Arrhythmia & 71 & & & & 0 \\
\hline 22 & M & No & No & No & $\mathrm{CV}, \mathrm{CP}$ & 70 & + & & & 0 \\
\hline 23 & M & No & No & Yes & Arrhythmia & 68 & + & & & 0 \\
\hline 24 & $\mathrm{M}$ & Yes & No & No & CD & 62 & + & + & & 3 \\
\hline 25 & $\mathrm{M}$ & No & No & No & Healthy & 51 & + & + & & 3 \\
\hline 26 & $\mathrm{M}$ & No & No & No & RS & 67 & + & & & 1 \\
\hline 27 & M & No & No & No & $\mathrm{CP}$ & 67 & & & + & 1 \\
\hline 28 & $\mathrm{M}$ & No & Yes & Yes & Healthy & 66 & & & & 0 \\
\hline 29 & M & No & Yes & No & Healthy & 60 & + & + & + & 3 \\
\hline
\end{tabular}

M, male; F, female; HTA, hypertension; CD, coronary disease; HC, hypercholesterolemia; AB, bronchial asthma; DM, diabetes mellitus; CMTD, Charcot-Marie-Tooth-disease; MBM, Mb menier; RS, rheumatoid spondylitis; CV, ventricular carcinoma; CP, prostate cancer.

samples were excluded from the comparisons. We divided Dupuytren's contracture-specific miRNAs into two categories based on detection calls in these patients' samples and the controls. In the first category, exclusively detected miRNAs were present only in Dupuytren's contracture patients and were absent from all controls. In the second category, preferentially expressed miRNAs were also detected in some controls, but were significantly overrepresented in Dupuytren's contracture patients $(q<0.05$; Fisher's exact test), and detected in at most $25 \%$ of the control samples. Detection calls were provided by the Agilent's Feature Extraction Software. In addition, significance of differential expression between the detected signals in two groups of samples was calculated by $t$-test; $q$-values provide an estimate of the false discovery rate among the most significant findings and were used to correct for multiple testing. ${ }^{22}$ Those miRNAs with $q<0.05$ in pairwise comparisons are considered differentially expressed.

None of the miRNAs were exclusively detected in control samples. To detect miRNAs with reduced expression levels in Dupuytren's contracture, we selected the miRNAs that had at least a twofold reduced mean expression level between the patient groups. For these miRNAs, significance of differential expression was estimated by $t$-test.

\section{mRNA Gene-Expression Profiling}

The mRNA-expression data we used was analyzed by Forsman et $a 1^{10}$ with patients similar to ours. They had 12 male Dupuytren's contracture patients, average age 59 (range 31-78 years). In all of these patients, the Dupuytren's contracture was considered to be mature and in the residual, advanced stage by the time of the operation.

The available data was reanalyzed with the GeneSpring (v.10) Software (Agilent Technologies). Fold changes and $t$-test were calculated for each of the over 20000 transcripts in Dupuytren's contracture patient samples and control samples following data filtration by expression.

\section{miRNA Target Prediction and Correlation with Gene-Expression Profile}

Target prediction was performed with TargetScan (http://www.targetscan.org/). Other web-based computational approaches including, miRanda (http:// www.microRNA.org) and Sanger miRBase (http:// www.microrna.sanger.ac.uk/targets/v5) also served for confirmation. With the use of GeneSpring (v.10) tools, the target gene list was translated to a geneexpression experiment, creating a new list from 
which the gene-expression levels of the deregulated miRNA target genes could be determined.

\section{Results}

\section{Identification of Dupuytren's Contracture-Specific microRNAs}

Our analysis revealed 45 Dupuytren's contracturespecific miRNAs detectable in $>50 \%$ of the Dupuytren's contracture patients, but in none of the fibroblast or fascia control samples. Two of the Dupuytren's contracture-specific miRNAs (miR-373 and miR-648) were detected exclusively in $>80 \%$ of the Dupuytren's contracture samples, and six additional miRNAs (miR-let-7d*, miR-205, miR-200a*, miR-34c-3p, miR-423-3p, and miR-551b*) were detected exclusively in $>70 \%$ of the Dupuytren's contracture samples $(q<0.05)$. We also identified 11 miRNAs, which were preferentially but not exclusively expressed in Dupuytren's contracture. These miRNAs were enriched in the patients, with a detection frequency of $>80 \%$ in Dupuytren's contracture, and of $<25 \%$ in the controls $(q<0.05$; Table 2).

In Dupuytren's contracture samples compared with control samples, 65 miRNAs were significantly downregulated $(q<0.05$; fold change $>2.0)$ (Table 3$)$.

In general, the differences in miRNA expression were greater between Dupuytren's contracture and fibroblast controls than between Dupuytren's contrac-

Table 2 Exclusively and preferentially expressed miRNAs ( $q$-value <0.05) in Dupuytren's contracture samples

\begin{tabular}{|c|c|c|c|}
\hline \multirow[b]{2}{*}{$\operatorname{miRNA}$} & \multirow[b]{2}{*}{$D C(\%)$} & \multicolumn{2}{|c|}{ Control } \\
\hline & & $(F i b / F a c)(\%)$ & Fib $(\%)$ \\
\hline \multicolumn{4}{|c|}{ Exclusively expressed } \\
\hline hsa-mir-648 & 86 & 0 & 0 \\
\hline hsa-mir-373* & 82 & 0 & 0 \\
\hline hsa-let-7d & 75 & 0 & 0 \\
\hline hsa-mir-205 & 75 & 0 & 0 \\
\hline hsa-mir-200a* & 72 & 0 & 0 \\
\hline hsa-mir-34c-3p & 72 & 0 & 0 \\
\hline hsa-mir-423-3p & 72 & 0 & 0 \\
\hline hsa-mir-551b* & 72 & 0 & 0 \\
\hline \multicolumn{4}{|c|}{ Preferentially expressed } \\
\hline hsa-mir-934 & 93 & 12.5 & 25 \\
\hline hsa-mir-23a* & 86 & 12.5 & 25 \\
\hline hsa-mir-617 & 86 & 12.5 & 25 \\
\hline hsa-mir-382 & 89 & 25 & 0 \\
\hline hsa-mir-498 & 86 & 25 & 0 \\
\hline hsa-mir-519e & 86 & 25 & 0 \\
\hline hsa-mir-584 & 86 & 25 & 25 \\
\hline hsa-mir-631 & 86 & 25 & 25 \\
\hline hsa-mir-181d & 82 & 25 & 0 \\
\hline hsa-mir-30b* & 82 & 25 & 0 \\
\hline hsa-mir-936 & 82 & 25 & 25 \\
\hline
\end{tabular}

*Indicates the mature miRNA is from the opposite arm of the precursor. DC, Dupuytren's contracture; Fib/Fac, fibroblast/fascia. ture and fascia (Figure 1); 68 miRNAs were expressed in $>80 \%$ of Dupuytren's contracture samples and were completely absent from fibroblasts.

\section{Relationship between miRNA and mRNA Expression}

MiRNAs are believed to regulate gene expression negatively at the level of mRNA transcription. ${ }^{23}$ We, therefore, examined whether any correlation existed between the expression profiles of miRNAs and their Dupuytren's contracture-associated target genes. After acquiring the differentially expressed miRNAs, we searched for their target genes using the web-based computational approaches, mainly the TargetScan. Those genes targeted by upregulated miRNAs in Dupuytren's contracture samples are expected to show under-expression compared with that of control samples and vice versa.

The data generally was in accordance with the hypothesis. Most Dupuytren's contracture-associated genes reported, including $W N T 5 A$, collagen synthesis-related genes, TGFB1, and $A D A M 12$, are over-expressed in patient samples, and our findings indicate that these are indeed mostly targeted by downregulated miRNAs (Table 4).

\section{Hereditary Trait, Recurrence Condition, and miRNA Expression}

Genetic susceptibility is an accepted factor that could lead to increased Dupuytren's contracture prevalence, but no statistically significant differences in miRNA expression were detectable between heredity/non-heredity or recurrent/non-recurrent groups $(q>0.05)$.

\section{Discussion}

MiRNAs are known as gene-expression regulators involved in many essential pathways. Our study of miRNA expression in 29 Dupuytren's contracture patients is, to our knowledge, the first report of its kind. Comparison was between miRNA-expression patterns of Dupuytren's contracture samples and of control samples represented by normal fascia and cultured fibroblast cells. An altered miRNA pattern could suggest involvement of miRNAs in the pathogenesis of Dupuytren's contracture.

Dupuytren's contracture has a characteristic miRNA pattern that distinguishes it from the control samples. This is evident in hierarchical clustering of the samples based on the miRNA detection calls (Figure 1). No specific or unique feature emerged among the patients to distinguish the minority of samples that closely resembled the control specimens from the majority of Dupuytren's contracture patients. However, 22 miRNAs were exclusively detected in the $>70 \%$ of the majority group of Dupuytren's contracture patients (group 1) 
Table 3 The 65 downregulated miRNAs in Dupuytren's contracture compared with controls

\begin{tabular}{|c|c|c|c|c|c|}
\hline $\operatorname{miRNA}$ & q-value $(<0.02)$ & $F C>2.0$ & $\operatorname{miRNA}$ & q-value $(<0.02)$ & $F C>2.0$ \\
\hline hsa-miR-133b & $1.78 \mathrm{E}-07$ & -31.94 & hsa-miR-365 & 0.002891372 & -3.52 \\
\hline hsa-miR-129-3p & 2.35E-07 & -18.9 & hsa-miR-21* & 0.002891372 & -3.79 \\
\hline hsa-miR-563 & 7.63E-07 & -20.03 & hsa-miR-933 & 0.00450724 & -2.61 \\
\hline hsa-miR-654-5p & $1.62 \mathrm{E}-06$ & -2.66 & hsa-miR-539 & 0.004734765 & -4.03 \\
\hline hsa-miR-129* & $2.90 \mathrm{E}-06$ & -19.48 & hsa-miR-1229 & 0.004738592 & -2.40 \\
\hline hsa-miR-92b & 2.90E-06 & -4.14 & hsa-miR-1238 & 0.004972111 & -2.43 \\
\hline hsa-miR-923 & 2.90E-06 & -6.87 & hsa-miR-301a & 0.004972111 & -3.58 \\
\hline hsa-miR-345 & 4.18E-06 & -3.1 & hsa-miR-130b & 0.004972111 & -2.19 \\
\hline hsa-miR-133a & 7.18E-06 & -7.44 & hsa-miR-10b & 0.00503656 & -4.72 \\
\hline hsa-miR-630 & $2.54 \mathrm{E}-05$ & -6.01 & hsa-miR-206 & 0.005616286 & -3.11 \\
\hline hsa-miR-22 & 3.62E-05 & -3.04 & hsa-miR-363 & 0.006404865 & -3.44 \\
\hline hsa-miR-222 & 7.60E-05 & -2.78 & hsa-miR-513b & 0.006404865 & -2.24 \\
\hline hsa-miR-339-3p & 7.60E-05 & -3.56 & hsa-miR-140-5p & 0.006732195 & -6.13 \\
\hline hsa-miR-494 & 0.000104812 & -6.43 & hsa-miR-126 & 0.0073314 & -4.78 \\
\hline hsa-miR-140-3p & 0.000104812 & -4.7 & hsa-miR-451 & 0.009336395 & -6.08 \\
\hline hsa-miR-135a & 0.000104812 & -4.43 & hsa-miR-100 & 0.009742879 & -2.53 \\
\hline hsa-miR-223 & 0.000160157 & -4.14 & hsa-miR-1 & 0.011527502 & -60.43 \\
\hline hsa-miR-1227 & 0.000160157 & -7.26 & hsa-miR-1234 & 0.011841197 & -2.06 \\
\hline hsa-miR-634 & 0.000194577 & -6.7 & hsa-miR-1236 & 0.012188798 & -3.63 \\
\hline hsa-miR-149 & 0.000265155 & -4.28 & hsa-miR-106b & 0.01391061 & -2.20 \\
\hline hsa-miR-422a & 0.000265155 & -2.4 & hsa-miR-143 & 0.01560635 & -4.36 \\
\hline hsa-let-7f-1* & 0.000265155 & -3.93 & hsa-miR-26a & 0.015660453 & -4.10 \\
\hline hsa-let-7b* & 0.000265155 & -3.92 & hsa-miR-26b & 0.016075024 & -5.28 \\
\hline hsa-miR-149 & 0.000265155 & -4.28 & hsa-miR-29c & 0.016440081 & -2.25 \\
\hline hsa-miR-425* & 0.000297306 & -3.94 & hsa-miR-30b & 0.017071422 & -3.90 \\
\hline hsa-miR-625* & 0.000366974 & -5.57 & hsa-miR-33b* & 0.017312469 & -2.17 \\
\hline hsa-miR-197 & 0.000502419 & -2.34 & hsa-miR-338-3p & 0.017312469 & -4.18 \\
\hline hsa-miR-766 & 0.000578821 & -2.59 & hsa-miR-520b & 0.017923984 & -2.23 \\
\hline hsa-miR-204 & 0.001211563 & -4 & hsa-miR-505 & 0.017923984 & -2.50 \\
\hline hsa-miR-1237 & 0.002025998 & -2.69 & hsa-miR-1225-3p & 0.019555786 & -2.06 \\
\hline hsa-miR-191* & 0.002298991 & -3.19 & hsa-miR-550 & 0.019567539 & -2.52 \\
\hline hsa-miR-1228 & 0.002457232 & -2.66 & & & \\
\hline hsa-miR-296-5p & 0.002834966 & -2.74 & & & \\
\hline hsa-miR-101 & 0.002891372 & -6.57 & & & \\
\hline
\end{tabular}

*Indicates the mature miRNA is from the opposite arm of the precursor.
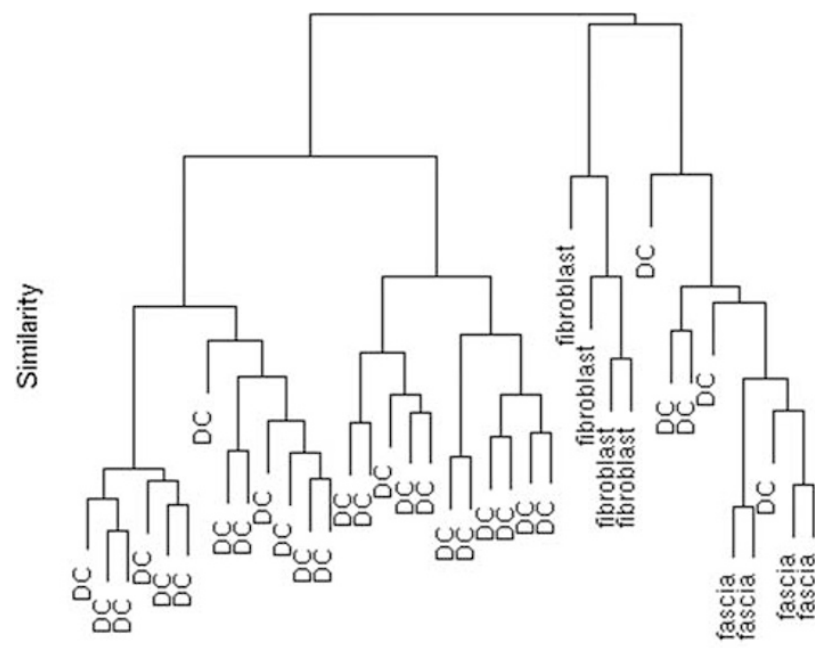

Patient samples

Figure 1 Hierarchical clustering of the samples based on miRNA detection calls. Note that the Dupuytren's contracture patients have a distinct expression profile that separates them from the fibroblast and fascia control samples.

compared with the minority group (group 2) (Table 5), whereas no exclusively detected miRNAs appeared in group 2 vs group 1.
Table 4 Target genes of downregulated miRNAs

Gene symbol miRNA

References

\begin{tabular}{lll}
\hline ADAM12 & miR-1, miR-101, miR-130b, miR-204, & 12 \\
& miR-206, miR-29c, miR-30b & \\
COL5A2 & miR-29c & 9 \\
TGFBI & miR-130b, miR-29c, miR-296-5p, & $35,37-39$ \\
& miR-301b, miR-654-5p & \\
MAFB & miR-130b, miR-29c, miR-301b & 9 \\
POSTN & miR-140-5p & $8,12,40,41$ \\
TNC & miR-1229, miR-1238, miR-494 & 12 \\
WNT5A & miR-22, miR-30b, miR-494 & 29,30 \\
ZIC1 & miR-101 & 32,33
\end{tabular}

It is also clear from these results that fibroblasts have a distinctly different miRNA-expression profile from those of from Dupuytren's contracture and fascia, one that can be attributed to the in vitro protocol and to the adaptation of such a growth environment. It is also becoming more evident that fibroblasts have an important part in the development of Dupuytren's contracture. ${ }^{24,25}$ All the main characteristics of Dupuytren's contracture, such as fibroproliferation and impaired wound-healing 
Table 5 The 22 miRNA exclusively detected in the majority of patients (group 1) compared with the minority (group 2) resembling the controls

q-value $(<0.05)$

\begin{tabular}{lcc}
\cline { 2 - 3 } miRNA & Group 1(\%) & Group 2 (\%) \\
\hline hsa-miR-181d & 100 & 0 \\
hsa-miR-30b* & 100 & 0 \\
hsa-miR-194* & 91 & 0 \\
hsa-miR-490-5p & 91 & 0 \\
hsa-miR-493 & 91 & 0 \\
hsa-miR-34c-3p & 87 & 0 \\
hsa-miR-423-3p & 87 & 0 \\
hsa-miR-583 & 87 & 0 \\
hsa-miR-138-2* & 79 & 0 \\
hsa-miR-200b* & 79 & 0 \\
hsa-miR-298 & 79 & 0 \\
hsa-miR-302C* & 79 & 0 \\
hsa-miR-501-3p & 79 & 0 \\
hsa-miR-516b & 79 & 0 \\
hsa-miR-616 & 79 & 0 \\
hsa-miR-500* & 75 & 0 \\
hsa-miR-615-3p & 75 & 0 \\
hsa-miR-10a* & 70 & 0 \\
hsa-miR-299-3p & 70 & 0 \\
hsa-miR-421 & 70 & 0 \\
hsa-miR-424* & 70 & 0 \\
hsa-miR-518e* & 70 & 0
\end{tabular}

*Indicates the mature miRNA is from the opposite arm of the precursor.

response, can be explained directly or indirectly by alterations of fibroblasts. ${ }^{26}$

Eight miRNAs were exclusively expressed $(>70 \%)$ in Dupuytren's contracture patient samples, but absent from all control samples, and 11 miRNAs were preferentially expressed $(>80 \%)$ in Dupuytren's contracture samples $(q<0.05$; Table 2); 65 miRNAs were downregulated in Dupuytren's contracture against all controls $(q<0.05$, FC $>2)$. These miRNAs may have a function in regulating the function of genes implicated in the pathogenesis of Dupuytren's contracture.

It is now clear that aberrant $\beta$-catenin signaling and its cellular accumulation can lead to various diseases including cancer and fibroproliferative diseases such as Dupuytren's contracture. ${ }^{26}$ Elevated levels of $\beta$-catenin have been reported in Dupuytren's contracture without involvement of $\beta$-catenin mutations. ${ }^{27}$ Possible mechanisms explaining $\beta$-catenin accumulation include alterations in the Wnt/ $\beta$-catenin signaling pathway, which makes it potentially the most important pathway involved in Dupuytren's contracture pathogenesis. This pathway is activated in the wound-healing process, recruiting proliferating fibroblasts to form a collagen matrix ${ }^{28}$ and it has an important function in regulating fibroblast proliferation and cell fate in various fibrous tumors. ${ }^{29}$ The Dupuytren's contracture-associated miRNAs identified here contribute to regulation of some of the genes related to this pathway, namely WNT5A (GeneID: 7474), ZIC1
(GeneID: 7554), and TGFB1 (GeneID: 7040). As can be seen in Table 4 and Figure 2, in Dupuytren's contracture, all of these genes are targeted by downregulated miRNAs, and their expression at mRNA level corresponds to negative regulation by these miRNAs.

O'Gorman et $a l^{30}$ noted non-involvement of the Wnt pathway in the activation of fibroblast and $\beta$-catenin accumulation in Dupuytren's contracture. Histologically, however, expression of cytoplasmic and nuclear $\beta$-catenin and Wnt5a, a member of the Wnt gene family, was observed by Degreef et $a{ }^{29}$ suggesting an upstream regulatory function for the Wnt pathway. WNT5A is among the target genes of miR-22, miR-30b, and miR-494. Furthermore, this protein has been shown to induce homodimerization and activation of the ROR2-receptor tyrosine kinase ${ }^{31}$ which is involved in signal transduction between cells and in the development of chondrocytes and cartilage. Forsman et $a l^{10}$ associated the upregulation of ROR2 with Dupuytren's contracture pathogenesis for the first time, using the geneexpression data reanalyzed in this study. The catenin (cadherin-associated protein) $\beta-1$ (CTNNB1 GeneID: 1499), which has been implicated in Dupuytren's contracture, ${ }^{27}$ is the target gene of several miRNAs, but its mRNA-expression level is not altered compared with that of control samples.

ZIC1, the target of miR-101, has been suggested to be an upstream regulator of several Wnt pathway genes including $W N T 1, W N T 4$, and $W N T 8 B,{ }^{32}$ and its expression has been described in fibroproliferative diseases including Dupuytren's contracture. ${ }^{33}$ Furthermore, Degreef et $a l^{34}$ reported on coexpression of ZIC1 and $\beta$-catenin in Dupuytren's contracture samples.

Several growth factors have been implicated in Dupuytren's development. ${ }^{35}$ TGF- $\beta$ (TGFB1) is thought to have a fundamental function in comparison with other growth factors, as it is the most abundant growth factor in Dupuytren's contracture, and also because of its central function in regulating cellular proliferation and differentiation and specifically in inducing fibroblast proliferation and extracellular matrix production. ${ }^{36}$ TGF- $\beta$ stimulation also induces elevation of $\beta$-catenin protein levels. ${ }^{37}$ In wound healing, TGF- $\beta$ is thought to function as a positive regulator of Wnt signaling in human dermal fibroblasts. ${ }^{38}$ Furthermore, in cultured fibroblasts from Dupuytren's contracture and patient-matched control samples, TGF- $\beta$ was noticed to stimulate collagen production. ${ }^{39}$ TGFB1 is the target of several downregulated miRNAs. Tenascin C (TNC, GeneID: 3371) and ADAM metallopeptidase domain 12 (ADAM12 GeneID: 8038) are involved in the TGF- $\beta$ pathway. ${ }^{12}$ Both were also over-expressed in our gene-expression analysis, which is consistent with the expression of the miRNAs that regulate these genes (Table 4). TNC is a Dupuytren's contracture-associated gene that is thought to be involved in the process of wound 


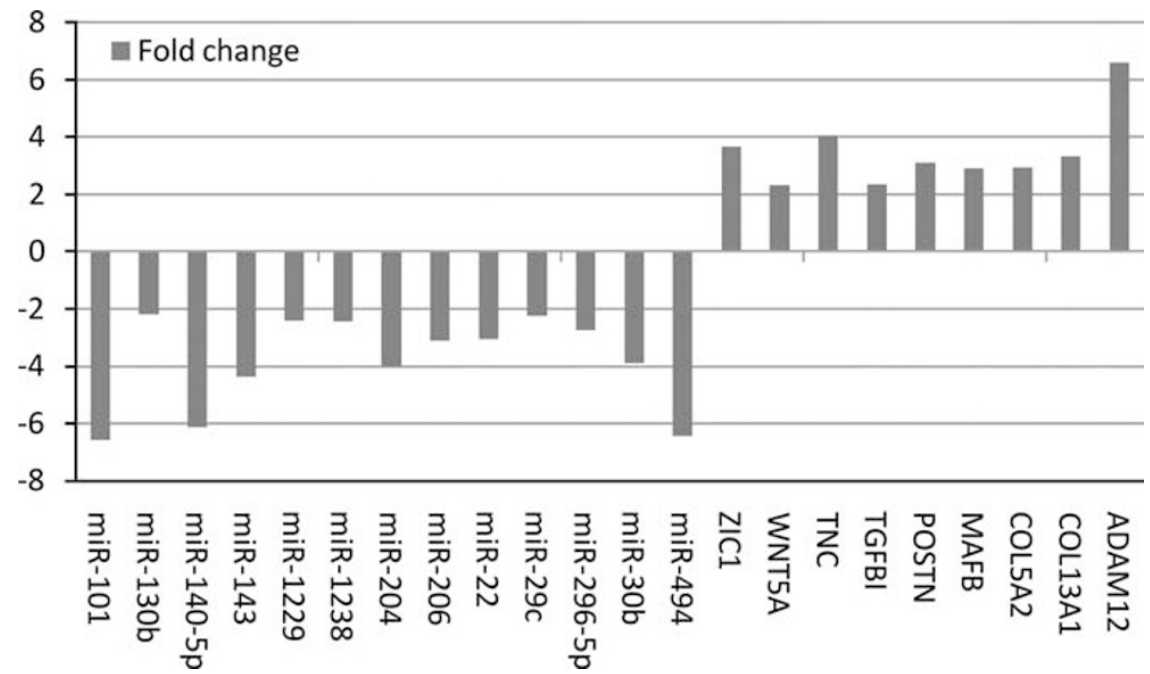

Figure 2 Downregulated miRNAs in Dupuytren's contracture and their over-expressed target genes, value = fold change.

healing and fibrosis, specifically in fibroblast and myofibroblast migration. ${ }^{12}$ In the study by Shih et al, TNC expression was associated with the proliferative nodules in Dupuytren's contracture.

Another over-expressed gene in Dupuytren's contracture targeted by downregulated miRNAs is Periostin osteoblast-specific factor (POSTN GeneID: 10631). Over-expression of this gene is associated with abnormal cell growth and with various cancers such as melanoma and oral and pancreatic cancers. ${ }^{8,12,40,41}$

In addition to abnormal proliferation of fibroblasts, Dupuytren's contracture is characterized by overproduction of collagen and extracellular matrix. Genes related to collagen synthesis (COL13A1, COL5A2), associated with Dupuytren's contracture in several studies, ${ }^{9,42}$ are targets of miR-29c.

Genes that have a function in cellular differentiation, development, and the ability to transform fibroblasts are regarded as the most important in the pathogenesis of Dupuytren's contracture. Our analysis recognized miR-130b, miR-29c, and miR-301b as regulating one such gene: $M A F B$ (GeneID: 9935). ${ }^{9}$ These miRNAs may contribute to the proper regulation and function of this important gene.

A huge variation exists in rate of recurrence of Dupuytren's contracture after surgery (2-63\%). ${ }^{43}$ The only usable method for predicting the rate of recurrence involves clinical staging methods based on epidemiological studies. Scoring systems are based on the personal history of the patient and severity of the contracture. ${ }^{44}$ Surveys suggest underlying molecular activity as responsible for recurrent Dupuytren's contracture. ${ }^{29}$ In our analysis, we compared patients with non-recurrent and recurrent Dupuytren's contracture, but detected no miRNAs with significant differential expression between the two patient groups $(q>0.05)$. This is in accordance with previous reports; Wilbrand et $a l^{45}$ observed that the presence of activation markers in connective tissue of Dupuytren's contracture was unrelated to disease recurrence, and Degreef et $a l^{29}$ observed no difference in $\beta$-catenin expression between Dupuytren's contracture that was recurrent or non-recurrent. It should be noted that larger and well-selected sets of patient samples might be needed to determine the differences. As Dupuytren's contracture is the most common heritable disorder of connective tissue, ${ }^{7}$ we analyzed patients with and without a history of familial Dupuytren's contracture, to elucidate possible differing patterns in miRNA expression between these two groups. No differentially expressed $(q>0.05)$ miRNAs between the two groups were, however, detectable. As in recurrency, larger samples might be necessary.

In summary, our findings suggest that miRNAs have a function in the pathogenesis of Dupuytren's contracture by regulating important genes of the $\beta$-catenin pathway. Identification of altered miRNA expression and targeted genes may thus provide the opportunity to develop molecular therapy strategies for treatment of Dupuytren's contracture. It should, however, be noticed that the data presented here is based on a relatively small number of samples and controls, and so further experiments are necessary. Further experiments could also look into whether similar miRNA-expression patterns exist in Dupuytren's contracture-related diseases such as plantar fibromatosis and Peyronie's disease.

\section{Acknowledgements}

We thank Professor Leif C Andersson from Department of Pathology, Haartman Institute, University of Helsinki, Finland for providing cultured fibroblast used in this study. This project was supported by grants from the Sigrid Jusélius Foundation, The Finnish Funding Agency for Technology and 
Innovation (TEKES, Grant number: 40101/07) and the special state subsidy research funds appropriated to the Helsinki and Uusimaa Hospital District (HUS EVO).

\section{Disclosure/conflict of interest}

The authors declare no conflict of interest.

\section{References}

1 McFarlane RM. Dupuytren's disease. J Hand Surg Ther 1997;10:8-13.

2 Shaw Jr RB, Chong AK, Zhang A, et al. Dupuytren's disease: history, diagnosis, and treatment. Plast Reconstr Surg 2007;120:44e-54e.

3 Ross DC. Epidemiology of Dupuytren's disease. Hand Clin 1999;15:53-62, vi.

4 Renard E, Jacques D, Chammas M, et al. Increased prevalence of soft tissue hand lesions in type 1 and type 2 diabetes mellitus: various entities and associated significance. Diabete Metab 1994;20:513-521.

5 Pan D, Watson HK, Swigart C, et al. Microarray gene analysis and expression profiles of Dupuytren's contracture. Ann Plast Surg 2003;50:618-622.

6 Bayat A, Walter J, Lambe $\mathrm{H}$, et al. Identification of a novel mitochondrial mutation in Dupuytren's disease using multiplex DHPLC. Plast Reconstr Surg 2005;115:134-141.

$7 \mathrm{Hu}$ FZ, Nystrom A, Ahmed A, et al. Mapping of an autosomal dominant gene for Dupuytren's contracture to chromosome $16 q$ in a Swedish family. Clin Genet 2005;68:424-429.

8 Qian A, Meals RA, Rajfer J, et al. Comparison of gene expression profiles between Peyronie's disease and Dupuytren's contracture. Urology 2004;64:399-404.

9 Lee LC, Zhang AY, Chong AK, et al. Expression of a novel gene, MafB, in Dupuytren's disease. J Hand Surg Am 2006;31:211-218.

10 Forsman M, Paakkonen V, Tjaderhane L, et al. The expression of myoglobin and ROR2 protein in Dupuytren's disease. J Surg Res 2008;146:271-275.

11 Satish L, Laframboise WA, O’Gorman DB, et al. Identification of differentially expressed genes in fibroblasts derived from patients with Dupuytren's Contracture. BMC Med Genomics 2008;1:10.

12 Shih B, Wijeratne D, Armstrong DJ, et al. Identification of biomarkers in Dupuytren's disease by comparative analysis of fibroblasts versus tissue biopsies in diseasespecific phenotypes. J Hand Surg Am 2009;34: 124-136.

13 Wurster-Hill DH, Brown F, Park JP, et al. Cytogenetic studies in Dupuytren contracture. Am J Hum Genet 1988;43:285-292.

14 Bonnici AV, Birjandi F, Spencer JD, et al. Chromosomal abnormalities in Dupuytren's contracture and carpal tunnel syndrome. J Hand Surg Br 1992;17:349-355.

15 Casalone R, Mazzola D, Meroni E, et al. Cytogenetic and interphase cytogenetic analyses reveal chromosome instability but no clonal trisomy 8 in Dupuytren contracture. Cancer Genet Cytogenet 1997;99:73-76.

16 Kaur S, Forsman M, Ryhanen J, et al. No gene copy number changes in Dupuytren's contracture by array comparative genomic hybridization. Cancer Genet Cytogenet 2008;183:6-8.
17 Kraljevic Pavelic S, Sedic M, Hock K, et al. An integrated proteomics approach for studying the molecular pathogenesis of Dupuytren's disease. J Pathol 2009;217:524-533.

18 Bartels CL, Tsongalis GJ. MicroRNAs: novel biomarkers for human cancer. Clin Chem 2009;55:623-631.

19 Winter J, Jung S, Keller S, et al. Many roads to maturity: microRNA biogenesis pathways and their regulation. Nat Cell Biol 2009;11:228-234.

20 Lu M, Zhang Q, Deng M, et al. An analysis of human microRNA and disease associations. PLoS One 2008;3:e3420.

21 Wang H, Ach RA, Curry B. Direct and sensitive miRNA profiling from low-input total RNA. RNA 2007;13: 151-159.

22 Storey JD, Tibshirani R. Statistical significance for genomewide studies. Proc Natl Acad Sci USA 2003; 100:9440-9445.

23 Fabbri M, Croce CM, Calin GA. MicroRNAs. Cancer J 2008;14:1-6.

24 Dave SA, Banducci DR, Graham III WP, et al. Differences in alpha smooth muscle actin expression between fibroblasts derived from Dupuytren's nodules or cords. Exp Mol Pathol 2001;71:147-155.

25 Moyer KE, Banducci DR, Graham III WP, et al. Dupuytren's disease: physiologic changes in nodule and cord fibroblasts through aging in vitro. Plast Reconstr Surg 2002;110:187-193; discussion 194-6.

26 Bowley E, O’Gorman DB, Gan BS. Beta-catenin signaling in fibroproliferative disease. J Surg Res 2007; 138:141-150.

27 Varallo VM, Gan BS, Seney S, et al. Beta-catenin expression in Dupuytren's disease: potential role for cell-matrix interactions in modulating beta-catenin levels in vivo and in vitro. Oncogene 2003;22: 3680-3684.

28 Moon RT, Kohn AD, De Ferrari GV, et al. WNT and beta-catenin signalling: diseases and therapies. Nat Rev Genet 2004;5:691-701.

29 Degreef I, De Smet L, Sciot R, et al. Beta-catenin overexpression in Dupuytren's disease is unrelated to disease recurrence. Clin Orthop Relat Res 2009; 467:838-845.

30 O'Gorman DB, Wu Y, Seney S, et al. Wnt expression is not correlated with beta-catenin dysregulation in Dupuytren's Disease. J Negat Results Biomed 2006;5:13.

31 Liu Y, Rubin B, Bodine PV, et al. Wnt5a induces homodimerization and activation of Ror2 receptor tyrosine kinase. J Cell Biochem 2008;105:497-502.

32 Merzdorf CS, Sive HL. The zic1 gene is an activator of Wnt signaling. Int J Dev Biol 2006;50:611-617.

33 Pourebrahim R, Van Dam K, Bauters M, et al. ZIC1 gene expression is controlled by DNA and histone methylation in mesenchymal proliferations. FEBS Lett 2007;581:5122-5126.

34 Degreef I, De Smet L, Sciot R, et al. Immunohistochemical evidence for Zic1 coexpression with betacatenin in the myofibroblast of Dupuytren disease. Scand J Plast Reconstr Surg Hand Surg 2009;43: 36-40.

35 Cordova A, Tripoli M, Corradino B, et al. Dupuytren’s contracture: an update of biomolecular aspects and therapeutic perspectives. J Hand Surg Br 2005;30: $557-562$.

36 Al-Qattan MM. Factors in the pathogenesis of Dupuytren's contracture. J Hand Surg Am 2006;31: 1527-1534. 
37 Cheon SS, Nadesan P, Poon R, et al. Growth factors regulate beta-catenin-mediated TCF-dependent transcriptional activation in fibroblasts during the proliferative phase of wound healing. Exp Cell Res 2004; 293:267-274.

38 Sato M. Upregulation of the Wnt/beta-catenin pathway induced by transforming growth factor-beta in hypertrophic scars and keloids. Acta Derm Venereol 2006;86:300-307.

39 Alioto RJ, Rosier RN, Burton RI, et al. Comparative effects of growth factors on fibroblasts of Dupuytren's tissue and normal palmar fascia. J Hand Surg Am 1994;19:442-452.

40 Bayat A, Stanley JK, Watson JS, et al. Genetic susceptibility to Dupuytren's disease: transforming growth factor beta receptor (TGFbetaR) gene polymorphisms and Dupuytren's disease. Br J Plast Surg 2003;56:328-333.
41 Lucas G, Brichet A, Roquelaure Y, et al. Dupuytren's disease: personal factors and occupational exposure. Am J Ind Med 2008;51:9-15.

42 Brickley-Parsons D, Glimcher MJ, Smith RJ, et al. Biochemical changes in the collagen of the palmar fascia in patients with Dupuytren's disease. J Bone Joint Surg Am 1981;63:787-797.

43 McFarlane RM, McGrouther DA, Flint MH. Dupuytren's Disease. Churchill Livingstone: New York, NY, 1990.

44 Dias JJ, Braybrooke J. Dupuytren's contracture: an audit of the outcomes of surgery. J Hand Surg Br 2006;31:514-521.

45 Wilbrand S, Flodmark C, Ekbom A, et al. Activation markers of connective tissue in Dupuytren's contracture: relation to postoperative outcome. Scand J Plast Reconstr Surg Hand Surg 2003;37:283-292.

46 Abe Y, Rokkaku T, Ofuchi S, et al. An objective method to evaluate the risk of recurrence and extension of Dupuytren's disease. J Hand Surg Br 2004;29:427-430. 